\title{
In-Hospital Outcomes of Acute ST Elevation Myocardial Infarction in Patients with Acute Kidney Injury
}

\author{
Md. Mosharul Haque ${ }^{1}$, M. Atahar Ali ${ }^{2}$, Mustafizul Aziz², Mohammad Ullah ${ }^{3}$, Mohammad Anowar \\ Hossain², Md. Shariful Islam², Abul Khair², Md. Rezawanul Islam ${ }^{4}$, Fahdia Afroz ${ }^{2}$, \\ Mohammad Ruhul Amin ${ }^{5}$, Kaniz Fatema Chowdhury ${ }^{6}$ \\ ${ }^{1}$ Upazilla Health Complex, Beanibazar, Sylhet, ${ }^{2}$ Department of Cardiology, NICVD, Dhaka, \\ ${ }^{3}$ Department of Cardiology, Col. Malek Medical College, Manikganj, ${ }^{4}$ DGHS, Mohakhali, Dhaka, \\ ${ }^{5}$ Shaheed Ziaur Rahman Medical College, Bogra, ${ }^{6}$ Department of Pathology, MAG Osmani \\ Medical College, Sylhet
}

Key Words:

Renal failure, IHD, Myocardial infarction

\begin{abstract}
Background: Acute kidney injury (AKI) is a risk factor for long-term adverse outcomes, including acute myocardial infarction and death. The objective of this study was to find out in-hospital outcomes in patients with acute ST elevation myocardial infarction with acute kidney injury.

Methods: A total 190 patients were included in this study and were equally divided into two groups, Group-I (with AKI) and Group-II (without AKI), according to absolute changes of serum creatinine level. AKI was defined as absolute changes in serum creatinine (SCr. at 48 hours' minus admission SCr) and categorized as mild AKI (increase of 0.3 to $<0.5 \mathrm{mg} / \mathrm{d}$ ), moderate AKI (increase of 0.5 to $<1.0 \mathrm{mg} / \mathrm{dl}$ ), and severe AKI (increase of e"1.0 mg/dl) using Acute Kidney Injury Network (AKIN) criteria.

Results: Overall in-hospital mortality rate was $14.7 \%$ in Group-I (mortality rate for those with mild, moderate, and severe AKI were 7\%, 13.3\%, and 31.8\%) compared with 5.3\% in Group-II. Regarding inhospital morbidities, significant arrhythmia (29.5\%) was the most common complication followed by acute heart failure (18.9\%), cardiogenic shock (12.6\%), and mechanical complications (4.2\%) which were more in Group-I compared to patients with Group-II. After adjustment of other risk variables, the multivariate logistic regression analysis revealed AKI remained an independent predictor of in-hospital mortality with adjusted odds ratios (OR) was 4.991 (95\% confidence interval, 1.873-13.301).

Conclusions: AKI is an independent predictor of in-hospital mortality and morbidity. It emphasizes the importance of efforts to identify risk factors and to prevent AKI during in-hospital management of acute STEMI patients.
\end{abstract}

(Cardiovasc. j. 2018; 11(1): 59-66)

\section{Introduction:}

Cardiovascular diseases (CVD) account for more than 17 million deaths globally each year. It contributes $30 \%$ of all deaths, $80 \%$ of those occur in low-income and middle-income countries. This figure is to grow to 23.6 million by the year 2030 . Coronary artery disease alone caused 7 million deaths worldwide in 2010. It is an increase of $35 \%$ since $1990 .{ }^{1}$ Estimates from the global burden of disease study suggests that by the year 2020 the South Asian part of the world (India, Pakistan, Bangladesh, Nepal) will have more individuals with atherosclerotic cardiovascular disease than any other region. ${ }^{2}$

CVD is the leading cause of morbidity and mortality throughout the world. Today CVD became the most common cause of death accounting for $30 \%$ of death worldwide, with $80 \%$ of the burden now occurring in developing countries. ${ }^{3}$ Coronary heart disease (CHD) is the single largest cause of death in the developed countries and is one of the leading causes of disease burden in developing countries as well. The exact prevalence of coronary artery disease in Bangladesh is not known. Only a limited number of small-scale epidemiological studies are available. The prevalence rate of coronary artery disease in Bangladesh is 3.4\% in rural population and $19.6 \%$ in urban population. ${ }^{4}$

Among the coronary heart disease, acute myocardial infarction (AMI) is one of the most common life threatening diagnoses among

Address of Correspondence: Dr. Md. Mosharul Haque, Upazilla Health Complex, Beanibazar, Sylhet, Bangladesh. Email: mosharulszmc2@gmail.com. 
emergency hospital admissions. Although the mortality rate after admission for myocardial infarction has declined by about $30 \%$ over the last two decades but it still remains high. Survival is markedly reduced in elderly patients (over age 70) whose in-hospital mortality rate is $21 \%$ as compared to $2.8 \%$ among patients 60 years old or younger. ${ }^{5}$

Myocardial infarction generally occurs when there is abrupt decrease in coronary blood flow following a thrombotic occlusion of a coronary artery previously narrowed by atherosclerosis. ${ }^{5}$ At present, STEMI comprises approximately $25 \%$ to $40 \%$ of $\mathrm{MI}$ in hospital presentations and in- hospital mortality approximately $5 \%$ to $6 \%{ }^{6}$

AKI is an important and common complication after acute myocardial infarction (AMI), affecting from 10 to $55 \%$ of the patients (this latter number referring to patients suffering from cardiogenic shock). ${ }^{7,8}$ AKI is the sudden deterioration of renal function characterized by elevated creatinine, reduced urine output, and retention of waste products. ${ }^{9}$ The development of AKI is associated with unfavorable outcomes and higher mortality after an AMI. 8, 10, 11 The mechanisms causing AKI in the first few days after an AMI are multifactorial, including systemic and renal hemodynamic changes secondary to an impaired cardiac output and an imbalance of vasodilators and vasoconstrictors and immunological and inflammatory kidney damage resulting from crosstalk between the heart and the kidney. ${ }^{12}$ Activation of neuro-hormonal systems such as the renin angiotensin-aldosterone system, increased catecholamine production, and endothelin elevation have been documented in patients with acute myocardial infarction. These neuro-hormonal alterations lead to renal arteriolar vasoconstriction and a reduction in renal perfusion pressure. ${ }^{10}$

A study reported that overall, 16.1\% had AKI, including $6.5 \%$ with mild AKI, $5.6 \%$ with moderate AKI, and $4.0 \%$ with severe AKI. ${ }^{11}$ In another study reported that AKI-associated mortality rate was 50\% in patients with STEMI complicated by cardiogenic shock, with inhospital mortality relative risk of $12.3{ }^{8}$ Similarly, worsening renal function was associated with an 11.4-fold increased risk of in-hospital mortality in patients who presented with STEMI, reported in a study. ${ }^{10}$

There is scarcity of literature that has systematically examined the association between AKI in STEMI patients with mortality and morbidity in-hospital setting. So, this study was designed to evaluate the in-hospital outcomes of acute ST elevation myocardial infarction in patients with acute kidney injury.

\section{Methods:}

This prospective cohort study was carried out among 190 patients with acute STEMI who received thrombolytic with Streptokinase (1.5 million units) within 12 hours of onset of chest pain with or without AKI in CCU of NICVD, Dhaka from February, 2016 to March, 2017. Acute STEMI patients who were not thrombolysis, subjected to do CAG, with previous $\mathrm{H} / \mathrm{O}$ percutaneous coronary intervention (PCI)/ CAG, coronary artery bypass grafting (CABG), MI, stroke or patients with known CKD or getting nephrotoxic drugs-like NSAID/ gentamycin, patient with acute heart failure or cardiogenic shock on admission or suspected myocarditis or pericarditis, valvular heart disease, congenital heart disease and cardiomyopathy were excluded from this study. Data collection was done after taking informed written consent from each patient or from legal guardian who fulfilled the criteria. Initial evaluation by meticulous history and detailed clinical examination was done and recorded in a pre-designed structured form. Demographic data (such as- age, sex), risk factors profile (including smoking, hypertension, diabetes, dyslipidemia and family history of coronary artery disease) and past medical history (including- prior MI, congestive cardiac failure, and stroke) were being noted.12 lead resting ECG (at a paper speed of $25 \mathrm{~mm} / \mathrm{sec}$ and $10 \mathrm{~mm} / \mathrm{mV}$ standardization) were done on admission, after thrombolysis, at discharge and when required. Blood sample were send for measurement of serum creatinine on the day of admission that was be considered as base line serum creatinine and at 48 hours' post-admission to identify AKI. Total 190 patients were divided into two groups, Group-I (Patients with acute STEMI with AKI=95) and Group- II (Patients with acute 
STEMI without AKI=95) according to inclusion and exclusion criteria. Then, patients with AKI were again subdivided into mild AKI ( $\mathrm{SCr}$ increaee, $0.3-<0.5 \mathrm{mg} / \mathrm{dL}$ ), moderate AKI ( $\mathrm{SCr}$ increase, $0.5-<1.0 \mathrm{mg} / \mathrm{dL}$ ), and severe AKI ( $\mathrm{SCr}$ increase, $\geq 1.0 \mathrm{mg} / \mathrm{dL}$ ) according to serum creatinine level. Results of other routine laboratory investigations were being noted. Echocardiography (by Siemens Acuson X700) was done to assess LV function within 48 hours of hospital admission and when needed. Ethical clearance was taken from ethical review committee, NICVD, Dhaka. Data were analyzed by using statistical package for continuous data was expressed as median or mean $\pm \mathrm{SD}$. Dichotomous data was expressed as percentage. Comparison of continuous variables was done by unpaired t-test or z-test as appropriate. Categorical data was analyzed with Chi-square $\left(\chi^{2}\right)$ test. The significance of the results as determined in 95\% confidence interval and a value of $\mathrm{P}<0.05$ was considered to be statistically significant. Multivariate logistic regression analysis was done to find out the predictors of in-hospital cardiac events.

\section{Results:}

The mean age of Group-I and Group-II studied patients were $55.4 \pm 7.8$ years and $47.5 \pm 7.0$ years respectively which were statistically significant $(p=0.001)$. Male patients were predominant in both groups with male female ratio was 6.9:1. Among the risk factors it was observed that hypertension, diabetes mellitus and dyslipidemia were significantly higher in Group-I patients than Group-II patients $(p<0.05)$. The mean level of baseline and peak serum creatinine in Group-I and Group-II were $(1.24 \pm 0.15 \mathrm{vs} .1 .07 \pm 0.25 \mathrm{mg} / \mathrm{dl})$ and $(2.07 \pm 0.59 \mathrm{vs}$. $1.21 \pm 0.25 \mathrm{mg} / \mathrm{dl}$ )and the mean difference between peak and baseline serum creatinine was $(0.83 \pm 0.63$ vs. $0.14 \pm 0.05 \mathrm{mg} / \mathrm{dl})$ which were statistically significant $(p=<0.001)$. According to the severity of AKI in Group-I study patients, $45.3 \%$ were mild, $31.6 \%$ were moderate and $23.2 \%$ severe AKI. The mean percent of ejection fraction was $47.0 \pm 7.4$ which was $45.7 \pm 6.3$ for the patients with Group-I and $50.3 \pm 5.8$ for the patients of Group-II. The mean duration of hospital stays of study population were $7.34 \pm 3.45$ days and 4.66 \pm 1.89 days in Group-I and GroupII patients respectively which was statistically significant $(p=0.01)$. Regarding to in-hospital outcomes it was observed that occurrence of significant arrhythmia (29.5\%) followed by acute heart failure (18.9\%), death (14.7\%), cardiogenic shock (12.6\%) and mechanical complications $(4.2 \%)$ and none of the patients suffered from stroke among both groups and it was evident that the proportion of in-hospital complications

Table-I

Demographic profile of study population $(N=190)$.

\begin{tabular}{lccc}
\hline & Group-I $(\mathrm{n}=95)$ & Group-II $(\mathrm{n}=95)$ & $\mathrm{p}$ value \\
\hline $\begin{array}{l}\text { Age in years (Mean } \pm \mathrm{SD}) \\
\text { (Range) }\end{array}$ & $55.4 \pm 7.8$ & $47.5 \pm 7.0$ & $0.001^{\mathrm{s}}$ \\
Gender & $(38-75)$ & $(34-66)$ & \\
Male & & & \\
Risk factors & $84(88.4 \%)$ & $82(86.3 \%)$ & $0.66^{\mathrm{NS}}$ \\
Smoking & $65(68.4 \%)$ & $53(57.9 \%)$ & $0.13^{\mathrm{NS}}$ \\
Hypertension & $51(53.7 \%)$ & $34(35.8 \%)$ & $\mathbf{0 . 0 1}^{\mathrm{S}}$ \\
Diabetes mellitus & $46(48.4 \%)$ & $32(33.7 \%)$ & $\mathbf{0 . 0 3} \mathrm{S}^{\mathrm{S}}$ \\
$\quad$ Dyslipidemia & $41(43.2 \%)$ & $30(31.6 \%)$ & $\mathbf{0 . 0 4} \mathrm{S}$ \\
$\quad$ Family H/O of CAD & $17(17.9 \%)$ & $15(15.8 \%)$ & $0.69^{\mathrm{NS}}$ \\
Pulse rate & $83.2 \pm 16.4$ & $75.7 \pm 8.1$ & $\mathbf{0 . 0 0 1} \mathrm{s}$ \\
(Mean \pm SD) & $(52-120)$ & $(60-94)$ & \\
Systolic blood pressure & $129.6 \pm 8.6$ & $122.2 \pm 7.5$ & $<\mathbf{0 . 0 4} \mathrm{S}$ \\
(mmHg) & $(110-140)$ & $(110-140)$ & \\
Diastolic blood pressure & $86.6 \pm 8.8$ & $77.5 \pm 6.1$ & $<\mathbf{0 . 0 3} \mathrm{S}$ \\
(mmHg) & $(70-95)$ & $(70-90)$ & \\
\hline
\end{tabular}

S - Standard Deviation, NS - Not Significant, S - Significant 
were significantly increased in Group-I patients than that of Group-II patients. When we observed the pattern of in-hospital complications according to the changes of serum creatinine, these complications were increased in frequency with increasing level of serum creatinine. Out of total 95 patients, $47.4 \%$ in Group-I and 21.0\%in Group-
II patients experienced adverse in-hospital outcomes which were statistically significant. The multivariate logistic regression analysis shows that the independent variables included DM, hypertension and AKI were statistically significant associated with in-hospital outcomes with ORs being 3.210, 2.236 and 4.991 .

Table-II

Distribution of the study population according to LVEF (N=190).

\begin{tabular}{|c|c|c|c|c|c|c|c|}
\hline \multirow[t]{3}{*}{ Ejection fraction (percent) } & \multicolumn{6}{|c|}{ Study patients } & \multirow[t]{3}{*}{$\mathrm{p}$ value } \\
\hline & \multicolumn{2}{|c|}{ Group-I (n=95 ) } & \multicolumn{2}{|c|}{ Group-II (n= 95) } & \multicolumn{2}{|c|}{ Total $(\mathrm{N}=195)$} & \\
\hline & Number & $\%$ & Number & $\%$ & Number & $\%$ & \\
\hline$<30$ (Severe dysfunction) & 0 & 0 & 0 & 0 & 0 & 0.0 & \\
\hline 30-44 (Moderate dysfunction) & 30 & 33.3 & 15 & 15.8 & 45 & 23.7 & \\
\hline 45-54 (Mild dysfunction) & 55 & 61.1 & 65 & 72.2 & 120 & 63.2 & \\
\hline$\geq 55$ (Normal) & 10 & 11.1 & 15 & 16.7 & 25 & 13.2 & \\
\hline Mean $\pm \mathrm{SD}$ (Range) & \multicolumn{2}{|c|}{$45.7 \pm 6.3(30-59)$} & \multicolumn{2}{|c|}{$50.3 \pm 5.8(43-66)$} & \multicolumn{2}{|c|}{$47.0 \pm 7.4(30-66)$} & $<0.49^{\text {ns }}$ \\
\hline
\end{tabular}

NS $=$ Not significant

Table-III

Baseline serum creatinine, peak serum creatinine and their difference status of the study population $(N=190)$.

\begin{tabular}{lccc}
\hline Variables & Group-I (n=95) & Group-II (n= 95) & p value \\
\hline & Mean \pm SD & Mean \pm SD & \\
Base line serum creatinine (mg/dl) at admission & $1.24 \pm 0.15$ & $1.07 \pm 0.25$ & $<0.001^{\mathrm{s}}$ \\
Peak serum creatinine (mg/dl) at 48 hours & $2.07 \pm 0.59$ & $1.21 \pm 0.25$ & $<0.001^{\mathrm{s}}$ \\
Difference (Peak - Baseline) $\mathrm{mg}$ & $0.83 \pm 0.63$ & $0.14 \pm 0.05$ & $<0.001^{\mathrm{s}}$ \\
\hline
\end{tabular}

$\mathrm{S}=$ Significant

Table-IV

Comparison of the study population according to hospital stay $(N=190)$.

\begin{tabular}{|c|c|c|c|c|c|}
\hline \multirow[t]{3}{*}{ Hospital stay (days) } & \multicolumn{4}{|c|}{ Study population } & \multirow[t]{3}{*}{$p$ value } \\
\hline & \multicolumn{2}{|c|}{ Group I (n=95) } & \multicolumn{2}{|c|}{ Group II $(\mathrm{n}=95)$} & \\
\hline & Number & $\%$ & Number & $\%$ & \\
\hline$<5$ & 57 & 60.0 & 85 & 89.5 & $<0.001^{\mathrm{s}}$ \\
\hline$\geq 5$ & 38 & 40.0 & 10 & 10.5 & $<0.001^{\mathrm{s}}$ \\
\hline Mean \pm SD & \multicolumn{2}{|c|}{$7.34 \pm 3.45$} & \multicolumn{2}{|c|}{$4.66 \pm 1.89$} & $<0.001^{\mathrm{s}}$ \\
\hline
\end{tabular}

$\mathrm{S}=$ Significant

Table-V

Distribution of Group-I study population according to AKI severity (N=95).

\begin{tabular}{|c|c|c|c|c|c|c|}
\hline \multirow[t]{2}{*}{ Number (Total) } & \multicolumn{2}{|c|}{ Mild AKI } & \multicolumn{2}{|c|}{ Moderate AKI } & \multicolumn{2}{|c|}{ Severe AKI } \\
\hline & No & $\%$ & No & $\%$ & No & $\%$ \\
\hline 95 & 43 & 45.3 & 30 & 31.6 & 22 & 23.2 \\
\hline
\end{tabular}


Table-V

Distribution pattern of in-hospital outcomes between two groups of study population $(N=190)$.

\begin{tabular}{lcccccc}
\hline In-hospital outcomes & \multicolumn{4}{c}{ Study patients } & \multirow{2}{*}{ p value } \\
\cline { 2 - 3 } & \multicolumn{2}{c}{ Group-I $(\mathrm{n}=95)$} & & \multicolumn{2}{c}{ Group-II (n=95) } & \\
\cline { 2 - 3 } & No & & & No & $\%$ & \\
\hline Significant Arrhythmia & 28 & 29.5 & & 9 & 9.5 & $<0.001^{\mathrm{s}}$ \\
Acute heart failure & 18 & 18.9 & & 6 & 5.7 & $0.009^{\mathrm{s}}$ \\
Cardiogenic shock & 12 & 12.6 & & 2 & 2.1 & $0.005^{\mathrm{s}}$ \\
Mechanical complications & 4 & 4.2 & & 1 & 1.1 & $0.17^{\mathrm{ns}}$ \\
Acute stroke & 0 & 0.0 & & 0 & 0.0 & \\
In-hospital death & 14 & 14.7 & 5 & 5.3 & $0.03^{\mathrm{s}}$ \\
\hline
\end{tabular}

NS $=$ Not significant $\quad \mathrm{S}=$ Significant

Table-VI

Distribution pattern of in-hospital outcomes according to the changes of serum creatinine in Group-I patients $(n=95)$.

\begin{tabular}{|c|c|c|c|c|c|c|}
\hline \multirow[t]{3}{*}{$\overline{\text { Outcome variables }}$} & \multicolumn{6}{|c|}{ Study patients } \\
\hline & \multicolumn{2}{|c|}{$\begin{array}{c}\text { Mild AKI } \\
(\mathrm{n}=43)\end{array}$} & \multicolumn{2}{|c|}{$\begin{array}{c}\text { Moderate AKI } \\
(\mathrm{n}=30)\end{array}$} & \multicolumn{2}{|c|}{$\begin{array}{c}\text { Severe AKI } \\
(n=22)\end{array}$} \\
\hline & No & $\%$ & No & $\%$ & No & $\%$ \\
\hline Significant arrhythmia & 10 & 23.3 & 8 & 26.7 & 10 & 45.5 \\
\hline Acute heart failure & 6 & 14.0 & 6 & 20.0 & 6 & 27.3 \\
\hline Cardiogenic shock & 3 & 7.0 & 4 & 13.3 & 5 & 22.7 \\
\hline Mechanical complication & 0 & 0.0 & 2 & 6.7 & 2 & 9.1 \\
\hline Acute stroke & 0 & 0.0 & 0 & 0.0 & 0 & 0.0 \\
\hline In-hospital death & 3 & 7.0 & 4 & 13.3 & 7 & 31.8 \\
\hline
\end{tabular}

Table-VII

Comparison of study population by adverse in-hospital outcomes (N=190).

\begin{tabular}{lcccccc}
\hline Adverse in-hospital & \multicolumn{2}{c}{ Group-I $(\mathrm{n}=95)$} & & \multicolumn{2}{c}{ Group-II $(\mathrm{n}=95)$} & $\mathrm{p}$ value \\
\cline { 2 - 3 } outcomes & Number & $\%$ & & Number & $\%$ & \\
\hline Present & 45 & 47.4 & & 20 & 21.0 & $<0.001^{\mathrm{s}}$ \\
Absent & 50 & 52.6 & & 75 & 79.0 & \\
\hline
\end{tabular}

Table-VIII

Multivariatelogistic regression analysis of in-hospital cardiac events with confounding factors $(N=190)$.

\begin{tabular}{lcccc}
\hline Variables of interest & $\begin{array}{c}\text { Standardized } \\
\text { coefficient }(\beta)\end{array}$ & Odds Ratio (OR) & 95\% CI of OR & $\mathrm{p}$ value \\
\hline Age & 0.005 & 1.005 & $0.420-2.405$ & $0.81^{\mathrm{ns}}$ \\
Smoking & 0.111 & 1.314 & $0.503-4.161$ & $0.59^{\mathrm{ns}}$ \\
Diabetes mellitus & 1.121 & 3.210 & $1.601-11.241$ & $0.005^{\mathrm{S}}$ \\
Hypertension & 1.022 & 2.236 & $1.239-3.401$ & $0.03^{\mathrm{s}}$ \\
Dyslipidemia & 0.322 & 1.537 & $0.316-2.787$ & $0.11^{\mathrm{ns}}$ \\
Family H/O of CAD & 0.144 & 1.145 & $0.377-2.714$ & $0.55^{\mathrm{ns}}$ \\
LVEF & 0.414 & 1.513 & $0.575-3.985$ & $0.40^{\mathrm{ns}}$ \\
Hospital stay> 5 days & 0.210 & 1.101 & $0.304-4.101$ & $0.48^{\mathrm{ns}}$ \\
AKI & 1.608 & 4.991 & $1.873-13.301$ & $0.001^{\mathrm{S}}$ \\
\hline
\end{tabular}

NS $=$ Not significant $\quad \mathrm{S}=$ Significant 


\section{Discussion:}

This prospective cohort study was carried out of 190 patients in the department of cardiology, National Institute of Cardiovascular Diseases from February 2016 to March 2017 to find out in-hospital outcomes of acute ST elevation myocardial infarction in patients with acute kidney injury.

The age and sex distribution of the studied patients were very close to other relevant studies. ${ }^{13,14}$ The mean age of the studied patients was $51.4 \pm 8.4$ years with mean age of the GroupI patients was $55.4 \pm 7.8$ years and Group-II patients was $47.5 \pm 7.0$ years. The mean age of Group-I was higher than Group-II which was statistically significant $(p=0.001)$. Male patients were predominant in study population $(87.4 \%)$ than female $(12.6 \%)$ with male female ratio being 6.9:1. In almost all studies related to coronary artery disease (CAD) similar male preponderance was found. As females are given less attention and access for them to the health care facilities is limited particularly in low socioeconomic population like our country may contribute for this male predominance.

This study found smoking as the most prevalent (63.2\%) risk factor for CAD. Among the other risk factors for CAD, the frequency of hypertension (44.7\%), diabetes mellitus (41\%), dyslipidemia (37.4\%)and family history of CAD (16.8\%).It was observed that hypertension, diabetes mellitus and dyslipidemia were significantly higher in Group-I patients than Group-II patients $(\mathrm{p}<0.05)$. Akanda, et al. ${ }^{13}$ found most prevalent risk factors were smoking (60\%) and dyslipidemia (60\%) followed by hypertension (35\%) and diabetes mellitus (10\%) in Bangladeshi population. Kumar, et al. ${ }^{15}$ found $40.4 \%$ were diabetic, $45.72 \%$ were hypertensive and family history of CAD were present $14.54 \%$ in an Indian population having AMI. In another study Parsa and Jahanshahi. ${ }^{16}$ found the prevalence of cigarette smoking, HTN, DM, hyperlipidemia, and family history of CAD were $45.8 \%, 39.1 \%$, $27.3 \%, 29.5 \%$, and $26.3 \%$ respectively. These differences might be due to variation in the life style, degree of motivation and level of education.
Among the clinical parameters including, the pulse rate $(83.2 \pm 16.4$ vs. $75.7 \pm 8.1$ per minute), systolic $(129.6 \pm 8.6 \mathrm{vs} .125 .2 \pm 7.5 \mathrm{mmHg})$ and diastolic $(86.6 \pm 8.8$ vs. $77.5 \pm 6.1 \mathrm{mmHg})$ blood pressure in between two groups were statistically significant $(p=0.001, p=0.04$ and $p=0.03$ respectively).

Average left ventricular ejection fraction (LVEF$\%)$ of the study population was 50.8 \pm 7.4 . LVEF

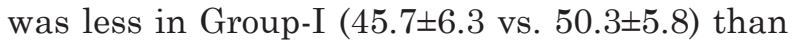
Group-II which was statistically non-significant. These findings were consistent with a study done by Marenzi, et al. ${ }^{8}$ with LVEF ( $32 \pm 10$ vs. $43 \pm 10$ ) between two groups. The reduced LVEF in AKI patients was related to development of more AHF.

Among study of serum creatinine, base line serum creatinine at admission, peak serum creatinine at 48 hours' post- admission and their difference in between two groups were statistically significant $(p=<0.001)$. It was also found that after analyzing of serum creatinine level, the STEMI patients in AKI group were categorized into mild, moderate and severe AKI of which $45.3 \%, 31.6 \%$ and $23.2 \%$ respectively. These findings were consistent with that of the findings of relevantstudies. ${ }^{10,11,17 .}$

In-hospital outcomes among the study population during their follow up period, in between two groups- in-hospital mortality was $(14.7 \%$ vs. $5.3 \%)$, significant arrhythmia was $(29.5 \%$ vs. $9.5 \%)$, acute heart failure was (18.9\% vs.5.7\%), cardiogenic shock was $(12.6 \%$ vs. $2.1 \%)$, mechanical complication was $(4.2 \%$ vs. $1.1 \%)$ and none of the study patients suffered from acute stroke. Among the individual outcomes, it was observed that significant arrhythmia, acute heart failure and cardiogenic shock were found to be significantly increased in number in between two groups $(p=<0.001, p=0.009, p=0.005$ respectively).

A study shown that a total 91 (9.4\%) deaths occurred in-hospital, with 47 (5.1\%) death occurring in patients with stable renal function and $44(44.9 \%)$ death in patients who developed worsening renal function, acute heart failure occurred $15 \%$ in non-AKI group and 33\% in moderate to severe AKI group. ${ }^{10}$ In an another 
study, ${ }^{18}$ it was found in STEMI patients, acute heart failure (Killip class II) was $22 \%$ and inhospital mortality was $8 \%$ in moderate to severe renal dysfunction. The increased mortality associated with impaired renal function was largely caused by events occurring in patients with AKI. In between two groups, Marenzi, et al. ${ }^{8}$ found ventricular tachycardia was (38\% vs. $36 \%)$, persistent tachycardia was (25\% vs. $11 \%)$, high grade AV block was (21\% vs. $13 \%)$ and mechanical complications $(2 \%)$ were same between two groups. These findings were consistent with our study population.

Regarding in-hospital outcomes of total patients, we found $(47.4 \%$ vs. $21 \%)$ in between two groups and the difference was statistically significant $(p=<0.001)$. These findings were supported by a study with total in-hospital outcomes (48.8\% vs. $2.1 \%) .{ }^{11}$

Among in-hospital death of STEMI patients, inhospital mortality rate was in mild, moderate and severe AKI were $7 \%, 13.3 \%$ and $31.8 \%$ respectively. It can be concluded that in-hospital mortality rate was statistically significant with increasing level of serum creatinine. Fox, et al. reported that in-hospital mortality rates for those with mild, moderate, and severe AKI were $6.6 \%, 14.2 \%$, and $31.8 \%$ compared with $2.1 \%$ in those without AKI. ${ }^{11}$ These findings were consistent with our study population. This is related to development of more complications in patients with AKI.

Among the status of hospital stay, it was observed that the duration of hospital stay was significantly prolonged ( $7.34 \pm 3.45$ vs. $4.66 \pm 1.89$ days; $\mathrm{p}=0.001$ ) in Group-I than Group-II patients. This finding were compatible with another study which was related to development of more complications in patients with AKI. ${ }^{8}$

In our study, after multivariate logistic regression analysis of odds ratio for characteristics of the subjects likely to cause adverse in-hospital outcomes, AKI emerged as a strong independent predictor of in-hospital outcomes (odds ratio: 4.991, 95\% confidence interval: $1.873-13.301, \mathrm{p}=0.001)$. In addition, DM (odds ratio: $3.210,95 \%$ confidence interval: 1.601 11.241, $\mathrm{p}=0.005$ ) and hypertension (odds ratio:
2.236, 95\% confidence interval: 1.239-3.401, $\mathrm{p}=0.03)$ also remained an independent, but weaker predictors, correlate of in-hospital outcomes. This result was compatible with a study done by Fox, et al. which shown that the ORs of diabetes mellitus and hypertension being 1.5(CI: 1.4-1.6) and 1.1 (CI: 1.1-1.2). ${ }^{11}$

We found that there was a variation of different outcomes in different studies as every patient respond to different stressful condition. 5, 7-10, 11, 17,18, 20,21 Some patients respond to exacerbated neuro-hormonal activation and others respond to more suppression of neuro-hormonal activation. Neuro-hormonal activation decreases progressively in post-infarction patients with good prognosis and inadequate suppression of neuro-hormonal activation may account, in part, for the poor clinical outcomes of patients manifesting acute kidney injury. Thus, AKI may serve as a simple marker of persistent neurohormonal activation.

Recently, the relationship between acute ST elevation myocardial infarction and acute kidney injury has gained greater recognition. No local study was done to assess the value of AKI in predicting in-hospital outcomes after acute STEMI patients. This study was designed to address the value of acute kidney injury as an indicator of in-hospital outcomes after acute STEMI patients as well as a small increase in serum creatinine leads to major in-hospital outcomes of those patients. It was found that a measure of AKI gave additional power to predict in-hospital events.

\section{Conclusion:}

From the results and observation of the study, it may be concluded that patients with acute ST elevation myocardial infarction who have acute kidney injury tend to be older, needed prolong hospital stay and associated with adverse inhospital outcomes than those without acute kidney injury.

\section{Recommendations:}

- Early detection, proper management and timely intervention strategy can be taken to reduce the morbidity and mortality of patients with acute ST elevation myocardial infarction with acute kidney injury. 
- As because no study has been carried out in this field in our country, we need to do much more studies to understand more clearly the relation between acute kidney injury of acute ST elevation myocardial infarction (STEMI) patients and in-hospital adverse outcomes in our socio-economic perspective of this country.

\section{Conflict of Interest - None.}

\section{References:}

1. Wong ND. Epidemiological studies of CHD and the evolution of preventive cardiology. Nature Reviews Cardiology 2014; 11: $276-289$.

2. Chakraborty B, Zaman F, Sharma AK. Combating Coronary Artery Disease in South Asia - What is special? Bangladesh Journal of Cardiology 2009; 01: 088- 090.

3. Chowdhury A W, Alam N, Khan HILR, Sabah KMN, Amin MG. The Pattern of Cardiac Disease at Coronary Care Unit of Dhaka Medical College Hospital. Cardiovasc j 2015; 7(2): 119-122.

4. Saquib N, Saquib J, Ahmed T, Khanam MA, Cullen MR. Cardiovascular diseases and Type 2 Diabetes in Bangladesh: A systemic review and meta-analysis of studies between 1995 and 2010. BMC Public Health 2012; 12: 434.

5. Shabbir M, Kayani AM, Qureshi O, Mughal MM. Predictors of fatal outcome in Acute Myocardial Infarction. J Ayub Med Coll Abbottabad 2008; 20(3): 14-16.

6. O'Gara PT, Kushner FG, Ascheim DD, Casey DE, Chung MK, Lemos JAD, et al. 2013 ACCF/AHA Guideline for the Management of ST-Elevation Myocardial Infarction: Executive Summary: A Report of the American College of Cardiology Foundation/American Heart Association Task Force on Practice Guidelines. J Am Coll Cardiol 2013; 61(4): 485-510.

7. Parikh CR, Coca SG, Wang Y, Masoudi FA, Krumholz HM. Long-term prognosis of acute kidney injury after acute myocardial infarction. Arch Intern Med 2008; 168(9): 987-995.

8. Marenzi G, Assanelli E, Campodonico J, Metrio M, de Lauri G, Marana I, et al. Acute kidney injury in ST-segment elevation acute myocardial infarction complicated by cardiogenic shock at admission. Critical Care Medicine 2010; 38(2): 438-444.

9. Warren J, Mehran R, Baber U, Xu K, Giacoppo D, Gersh $\mathrm{BJ}$, et al. Incidence and impact of acute kidney injury in patients with acute coronary syndromes treated with coronary artery bypass grafting: Insights from the Harmonizing Outcomes With Revascularization and Stents in Acute Myocardial Infarction (HORIZONS-AMI) and Acute Catheterization and Urgent Intervention Triage Strategy (ACUITY) trials. Am Heart J 2016; 171(1): 40-47.

10. Goldberg A, Hammerman H, Petcherski S, Zdorovyak A, Yalonetsky S, Kapeliovich M, et al. In hospital and 1-year mortality of patients who develop worsening renal function following acute ST-elevation myocardial infarction. Am Heart J 2005; 150(2): 330-337.

11. Fox CS, Muntner P, Chen AY, Alexander KP, Roe MT, Wiviott SD. Short-term outcomes of acute myocardial infarction in patients with acute kidney injury: a report from the national cardiovascular data registry. Circulation 2012; 125(3): 497-504.

12. Ronco C, Haapio M, House AA, Anavekar N, Bellomo R. Cardiorenal syndrome. J Am Coll Cardiol 2008; 52(19): 1527-1539.

13. Akanda MAK, Ali SY, Islam AEMM, Rahman MM, Parveen A, Kabir MK, et al. Demographic profile, clinical presentation \& angiographic findings in 637 patients with coronary heart disease. Faridpur Medical College Journal 2011; 6(2): 82-85.

14. Gupta S, Gupta VK, Gupta R, Arora S, Gupta V. Demographic profile and prevalence of risk factors and their correlation with STEMI, NSTEMI and premature CAD in documented CAD patients. Journal of Preventive Cardiology 2012; 1(4): 164-172.

15. Kumar N, Sharma S, Mohan B, Beri A, Aslam N, Sood N, et al. Clinical and angiographic profile of patients presenting with first acute myocardial infarction in a tertiary care center in northern India. Indian Heart Journal 2008; 60: 210-214.

16. Parsa AFZ. Jahanshahi B. Is the relationship of body mass index to severity of coronary artery disease different from that of waist-to-hip ratio and severity of coronary artery disease? Paradoxical findings. Cardiovascular Journal of Africa 2015; 26(1): 13-16.

17. Pimenta E, Ramos RF, Gun C, Santos ES, Timerman A, Piegas LS. renal function outcome in acute myocardial infarction as a prognostic factor of in-hospital events and at 0ne-year follow-up .Arquivos Brasileiros de Cardiologia, 2006; 86(3): 170-174.

18. Santopinto JJ, Fox KAA, Goldberg RJ, Budaj A, Piñero G, Avezum A, et al. Creatinine clearance and adverse hospital outcomes in patients with acute coronary syndromes: findings from the global registry of acute coronary events (GRACE).Heart 2003; 89: 1003-1008.

19. Cannon C, Brindis R, Chaitman B, Cohen D, CroSYNTAX J, Drozda J, et al. 2013 ACCF/AHA Key Data Elements and Definitions for Measuring the Clinical Management and Outcomes of Patients With Acute Coronary Syndromes and Coronary Artery Disease: A Report of the American College of Cardiology Foundation/American Heart association Task Force on Clinical Data Standards (Writing Committee to Develop Acute Coronary Syndromes and Coronary Artery Disease Clinical Data Standards). J Am Coll Cardiol 2013; 61(9): 992-1025.

20. Ghaffari S, Separham A, Pourafkari L. Association of electrocardiographic changes with severity of coronary artery disease and short term outcome in patients with non-ST-segment elevation acute coronary syndromes, Saudi Medical Journal 2010; 31(4): 400-405.

21. Goldberg A, Kogan E, Hammerman H, Markiewicz W, Aronson D. The impact of transient and persistent acute kidney injury on long-term outcomes after acute myocardial infarction. Kidney International 2009; 76(8): 900-906. 